\title{
The Role of MEG in Unveiling Cognition
}

\author{
Silva Nunes M.V. ${ }^{1}$, Maestú F. ${ }^{2}$ and Castro Caldas A. ${ }^{1}$ \\ ${ }^{1}$ Institute of Health Sciences of The Catholic University of Portugal, \\ ${ }^{2}$ Laboratory of Cognitive and Computational Neuroscience, Centre for Biomedical \\ Technology, Complutense University of Madrid \\ ${ }^{1}$ Portugal \\ 2Spain
}

\section{Introduction}

As Dolan (2008) has suggested, to measure brain activity associated with discrete states of mind is the Holy Grail of cognitive neuroscience. Fortunately, and as pointed by several authors, the techniques and analitic tools to study the living brain have greatly evolved. In fact, since 1970 there have been remarkable advances in the way we study human brain thought imaging and, as Raichle (2001) has pointed out, the results of this work constitute a strong argument for the development of new imaging methods to try to overcome the weakness of data gathering or data analysis. In conjunction, new hardware and signal processing methods, are also addressing the weaknesses of data gathering and data analyses.

In his 1998 book, Papanicolau compares imaging with the process of taking photographs and makes the argument that all imaging is mediated rather than direct. Whether one is talking about photography or brain imaging, all images are partial, not complete models of the reality imaged, and the accuracy with which they represent reality varies. Though it might seem obvious, it is important to reinforce that all imaging is mediated rather than direct, in order to emphasize that although one of MEG's advantages is that it "measures directly" brain activity, as with every other technique, the obtained imaging is mediated by processes of recording and registration of a form of energy and processes of development or construction of a representation or a model of the object. Because virtually an infinity of different models can be constructed based on the same reality, functional brain imaging is a "mixed blessing: it renders functional imaging valuable and promises tools for new discoveries, while it entails the perils of deception, error and disappointment" (Papanicolau, 1998).

Regarding the importance that imaging now has in the study of cognition, it is also worthwhile to note that sometimes the advances were not only methodological. For instance, $f$ MRI, in comparison with PET: "democratized access to a powerful technology for investigating the living human brain, allowing a broad cross-section of academic disciplines to pursue new agendas" (Dolan, 2008). These disciplines, like cognitive psychology or neuropsychology, with their specific contributions, brought to the fore questions related to human cognition in the development of the field of neuroimaging. The same is happening with MEG. 


\section{Studying cognition}

Cognition can be understood not as a single process, but as several performances and behaviors in a well designed task or in daily living activities that always come from the activity of the brain (Dixon \& Nilsson, 2004). Therefore, it seems possible to clearly state the task of functional brain imaging as: identifying regions and their temporal relationships associated with the performance of a well designed task (Raichle, 2001).

However, there are several caveats in this approach. Brain activation patterns captured by functional brain imaging are assumed to reflect aspects of neuropshysiological mechanisms necessary to a function. If we understand a function as a process of production of a set of similar phenomena that serve a common purpose or accomplish a common goal, and consider each phenomenon in the set as a token or an instance of the function, then when we are studying cognition we must provide a way to trigger the phenomena tokens of the function, the stimulus and instructions that constitute the experimental task (Papanicolau, 1998). However, tasks are never pure and always involve a complex blend of different cognitive operations. In functional neuroimaging studies this implies that the set of brain regions associated with a particular task may reflect not only the cognitive operation that we are interested in studying but any of the cognitive operations engaged by the task.

Several approaches have been used to tackle this difficulty. First with PET, neuroimaging was based in subtraction techniques derived from a concept from Donders (see Raichle, 2001, for a more detailed description). Here different blocks of stimuli were presented to subjects, one block representing the condition of interest (task state) and another block associated with a control condition (control state). In the beginning of $f$ MRI these block designs were also common. (Aguirre \& D'Esposito, 2000; Donaldson \& Buckner, 2001). However, as suggested by Stufflebeam and Rosen, with the implementation of event-related fMRI, a procedure similar to the measuring of evoked responses in electrophysiology (Aguirre \& D'Esposito, 2000; Donaldson \& Buckner, 2001), it was possible to overcome the limitations of a more rigid block design and, at same time, use the knowledge of the properties of neurons to enhance both spatial and functional sensitivity (Stufflebeam and Rosen, 2007).

In the modern imaging methods currently available, functional magnetic resonance imaging (fMRI) is still one of the most frequently used techniques. Many investigators have aimed at localizing function, identifying the brain areas that are responsible for cognitive tasks. However, these techniques have not clearly identified which properties of the brain are responsible for cognition (Douw et al., 2011).

As Dolan (2008) points out, although many of the findings resulting from the extensive study of cognition by means of neuroimaging reproduced what was discovered using lesion-based models, there were also unexpected results. According to Dolan, these findings have to do with the fact that sophisticated perspectives on the impact of a lesion suggest that cognition arises not just out of functional differentiation, but also from functional integration. This implies that the impact of a localized lesion is likely to reflect both local and distributed effects, evidenced by more widespread activations seen with neuroimaging than was predicted by a lesion-deficit model. Besides that, cognitive functions require precise coordination of neural activity across long distances in the brain (Stufflebeam \& Rosen, 2007). 


\section{Why use MEG to study cognition?}

Each of the several available imaging techniques has its own individual strengths and weaknesses. Some are particularly useful in temporal resolution (generally understood as the smallest time period of brain activation that can be distinguished), others in spatial resolution (generally understood as the smallest area of brain activation that can be distinguished). The electromagnetic imaging techniques include MEG and EEG, and they might not be thought of as imaging at all (Stufflebeam \& Rosen, 2007). However, they possess excellent temporal resolution since they measure the underlying neural currents directly (although requiring the processes of acquisition and development) on the order of milliseconds, albeit with limited spatial resolution.

Hari et al. (2000) suggest that this is a major advantage since the brain is a real-time processor, which implies that its functions can best be studied with tools that allow tracking of neural activation within the millisecond time scale. Only EEG and MEG are capable of providing information at such a high temporal resolution, and in a non-invasive manner, necessary for the study of brain dynamics. According to Stam (2005), two major sources for a renewed interest in MEG and EEG can be identified as "1) the realization that a full understanding of the neuropshysiological mechanisms underlying normal and disturbed higher brain functions cannot be derived from a purely reductionist approach and requires the study of emergent phenomena such as large scale synchronization of neuronal networks in the brain 2) the introduction of new techniques, concepts and analytical tools which make it possible to extract more and more meaningful information from recordings of brain activity." A cited example of these analytical tools is the application of time series analysis techniques derived from nonlinear dynamics to the study of MEG and EEG. Although it lies outside the scope of this chapter, it is nevertheless important to understand that nonlinear time series analyses, when applied to time series of brain activity, allow us to better understand the dynamics of the underlying system. This is particularly true at the level of functional connectivity, a concept referring to any type of correlation between time series of brain activity. The underlying assumption is that functional connectivity reflects, at least to some extent, functional interactions between different brain regions (see Stam, 2005 for a detailed description), and this represents a major step forward, passing from localization to connectivity.

There are several detailed descriptions of the underlying phenomena measured by MEG. It is usually said that MEG measurements are sensitive to postsynaptic currents in the dendrites of cortical pyramidal neurons (Hämäläinen et al., 1993). These changing synaptic currents of cortical pyramidal neurons give rise not only to an electric field but also to a magnetic field, since changes in an electric field induce a magnetic field. When a large set of cells that are typically not synchronized begin to signal in unison, their combined electric currents will create a large deviation. With MEG we record the surface distribution, that is the magnetic flux or the magnetic fields, that arises from the primary or source currents overcoming the EEG limitations since, unlike with electrical signals, tissues do not distort magnetic signals. MEG can be used to detect spontaneous as well as evoked brain activity (see Hari, 1998; Hämäläinen and Hari, 2002; \& Papanicolau, 1998 for detailed descriptions).

In a review article Zamrini et al. (2011) identify other advantages of MEG, specifically over other measures of electrical activity of the brain, including its freedom from the requirement 
for a reference electrode which has the potential to improve calculations of the electrical power and source localization of the signal. Also, because MEG normally uses many more detectors than traditional EEG, it allows for better spatial resolution. Because there is no need for a reference point in MEG studies, this facilitates synchronization and coherence analyses, which is increasingly important in order to understand the relations between brain and cognition. Another important factor in the study of cognition is that MEG is more sensitive to the gamma band and, as pointed out by Zamrini (2011), there is increasing evidence of the relations between gamma band and cognition. Hari et al. (2000) identify other specific advantages of MEG in the study of human brain functions, reinforcing that it is noninvasive, with excellent temporal resolution, reflecting neural activation directly (mainly postsynaptic currents), and it is selective to activation of fissural cortex which is difficult to access with other techniques. Another important claim is that MEG allows the study of individual processing strategies because conclusions can be made on the basis of a single subject. This is particularly important because each brain is unique, and the active areas can differ between individuals in similar tasks, with the expectation that these differences can become clearer in the timing than in the sites of cortical activation (He \& Liu, 2008). This further reinforces the unique contribution made by MEG to the understanding of cognitive strategies, in normal as well as in clinical populations. Hari et al. (2000) also mention that MEG does not require subtraction between conditions, which makes cognitive experiments more flexible.

Synthesizing MEG is appropriate for studies of fast activation sequences of human cortical activation. It also allows comparisons of timing between distinct brain regions, being capable of capturing small time differences. In fact, as pointed out by several authors in several studies, MEG can detect fast switching on the different brain regions as they become active with a time resolution in the order of ms.

However, as with all the other techniques MEG has some disadvantages. The recorded magnetic field is much more difficult to measure than the electric currents recorded by EEG, particularly because they are of very low field strength when compared to the surrounding magnetic fields, namely the magnetic field of the earth. This implies that in order to be able to capture these magnetic distributions several technological advances were needed and a very expensive apparatus is required. In Stam's description the requirement is for "Cooled conductive coils in order to make them superconductive which allows the current to flow without resistance. Magnetic fields can induce currents in theses coils, and thanks to superconductivity very weak magnetic fields can be measured. Converting the currents in the coils to a signal that can be measured requires the SQUID (superconducting quantum interference device): very small rings with a very small interruption which can be crossed by electrons thanks to the quantum effects. SQUIDS are the key elements in the MEG system. Further improvement of signal-to-noise ratio is achieved by measuring only local gradients (gradiometers) instead of the absolute field. Finally the whole MEG recording system is shielded from the magnetic fields of the surroundings by placing it in a magnetically shielded room (MSR) .... The stimulation apparatus that gives rise to electromagnetic fields has to be placed outside the room and has to be transferred to the subject inside the MSR in an appropriate way" (see Stam, 2010). All of this apparatus is responsible for one of its major drawbacks, the price. It also requires the subject to cooperate and to keep the head immobile during the recording, which makes it difficult to use this technique with all populations, as it will be important for a range of cognitive studies. Another often pointed disadvantage is 
that deep and radial sources are largely neglected since magnetic flux deriving from deep sources will reach the head surface with difficulty and those derived from the radial source will not be captured.

Besides that, the data interpretation on MEG is hampered by the non-uniqueness of the inverse problem. As stated previously, MEG field patterns recorded outside the head are interpreted in terms of current distribution in the brain. The characteristics of the source inside are estimated on the basis of the regular features of the recorded distribution. This solution results in a hypothetical distribution. From perfectly known phenomena are derived the imperfectly known causes of the phenomena (the five parameters of the source, strength, orientation and location, X, Y, Z) (see Papanicolau, 1998). As suggested by Hauk (2004), several efforts have been made to tackle this problem, but explicitly or implicitly it is recognized that according to the Helmholtz principle the bioelectric inverse problem has no unique solution. In fact, whatever result is obtained with one method there are still infinitely other possible solutions equally compatible with the recorded signal, which implies the question: Which method yields the correct answer for the bioelectromagnetic inverse problem? In Hauk's opinion this is a futile question: "it's like reconstructing a threedimensional object from its shadow because those data simply don't exist."

Aside from these difficulties, and in general, MEG, with its ability to follow the course time of brain activation, has been proven increasingly useful in the study of cognition. Several advances are being made in the understanding of oscillatory and synchronized brain activity and it is now assumed that synchronization of neural activity between different brain regions may reflect functional interactions between these regions, which will reinforce the ability of MEG to respond to the new focus on functional neuroimaging - no longer where but how.

\section{Memory and MEG}

Because of its role in cognitive functioning, and by the salience of its change across a lifetime with normal as well with pathological aging, memory has been a field of interest for neuroimaging, and MEG is no exception. As we shall see, because it is of extreme importance to implement techniques that are able to distinguish normal brain aging from abnormal and degenerative processes as early as possible, there is a great amount of work being done that addresses memory networks in pathological groups. However, there are also several projects trying to apply MEG techniques to a better understanding of the organization of memory networks. For example, MEG has been used to better understand the relative contributions and relations among different memory systems. Standing beyond the scope of this brief review concerning memory, since the seminal work of Scoville and Milner (1957) two areas have been consistently associated with memory processing, namely the medial temporal lobe (MTL) and the prefrontal cortex (PFC), with the medial temporal lobe being consistently associated with long-term memory, and the prefrontal being associated with working memory. However, with the advancement of imaging techniques there is growing support for the hypothesis that both structures are part of a unified memory network, challenging a more localizationist and segregated approach. As pointed out by Anderson et al. (2010), although functional connectivity analysis of fMRI data has revealed correlations between the lateral PFC and MTL in several types of memory, hemodynamic data does not provide an insight into the underlying neuronal processes. Using implanted electrode grids, these authors showed that the values of theta coherence 
between these two brain areas are task modulated, which in their opinion constitutes evidence of MTL-PFC interaction during memory tasks and points to the relevance of the role of theta activity in mediating this interaction. The point here is that previous results, specifically with MEG, found support for the idea that the increase of working memory load can lead to the increase of functional connection of extended regions (Tanaka et al., 2003). Results with MEG seem to reinforce the concept of a neural network shared by both types of memory (Campo et al., 2005). In a recent study of working memory, where MEG signals were recorded while subjects were performing a verbal working memory task with parametric load manipulation, results led the authors to propose an alternative principle of theta functionality, showing that the oscillatory mechanisms of verbal working memory comprise interacting processes across a distributed network and, more importantly for the present point, that capacity limitations are associated with both increased and decreased frequencies in the 4-12 hertz range (Moran et al., 2010).

In terms of the role of MEG in studying pathological memory networks, the great bulk of the research has been on Alzheimer's disease (AD). Alzheimer's disease (AD) is one of the major concerns of developed nations, being frequently cited as the main drawback of the advance in life expectancy. In fact, the incidence and prevalence of AD rises as individual's age. On the other hand, because of the nature of the signal measured by MEG the neurons affected by the pathological processes underlying AD can be measured directly. In concordance with this, and as summarized by Rossini et al. (2007), different types of neuropshysiological techniques have demonstrated abnormalities in AD during memory tasks, namely hyperactivation of prefrontal and midline activity, functional disconnection between prefrontal cortex and hippocampus, and stronger activation of the left temporal cortex, concluding that there is evidence for the importance of neuroelectric measurements in AD. In a MEG study examining focal slow wave generators they were found to be abnormally frequent in the cortical structures, particularly in the parietal and temporal lobes of patients with an AD diagnosis. The authors interpreted such an enhanced density of focal slow wave generators as the functional counterpart of the neurodegenerative process associated with the disease. The AD patients exhibited more MEG delta and theta activity than healthy aged control subjects because the focal slow waves would be focused on temporal and parietal structures, which is in accordance with previous results using EEG analyses. They also found that temporoparietal dysfunction can predict the cognitive and functional status of patients with AD (Fernandez et al., 2002).

It is known that physiological aging affects resting, but also event-related alpha rhythms. In a MEG study specifically addressing event-related desyncronization (ERD) in demented patients (compared with a group of normal young people and a group of normal elderly) in a two-task paradigm with different loads, it was shown that during the delay period of both tasks, the alpha ERD was stronger in demented patients than in the other groups (Babiloni et al., 2005). This suggests an abnormal increase of cortical excitation or disinhibition in dementia, generally compatible with differences in neurotransmission systems, namely at the cholinergic level. It is interesting to note that MEG in AD seems to be sensitive to changes in early cortical processing. For instance, in a study aimed at investigating the auditory-steady state response (SSR) in mild to moderate non-medicated AD patients as recorded by MEG it was found that the amplitude of auditory SSR is significantly increased in $\mathrm{AD}$ as compared to normal controls. This is interpreted by the authors as an impaired adaptation of auditory neurons to repetitive stimuli and decreased inhibition, probably due to changes in the acetylcholine system. These results seem then to reflect changes in the 
primary cortex, showing that stimuli deficit processing can be present at an early cortical stage (Osipova, 2006).

Although the majority of the conditions, and Alzheimer's disease in particular, are determined by several interrelated factors, it is also true, and a result of several constraints, that the majority of the studies rely on a single technique. As pointed out by Fernandez at al. (2003) in a brief review, each technique has been able to identify features of AD. For instance, several studies using metabolic methods, namely PET and SPECT, evidenced features of $\mathrm{AD}$, particularly hipometabolism-hipoperfursion in the temporoparietal and hipocampal. Similarly, with EEG an increase in slow activity, usually focused in the temporoparietal regions, together with decreased activity in the alpha band are considered the prototypical features of AD. Structural volumetric measurements obtained a reduced volume of temporaparietal and hipocampal formation volume, etc. The point the authors make is that magnetic source imaging can overcome some difficulties of previous approaches combining high temporal and high spatial resolution in detecting focal neural activity. One important aspect when using any imaging method is the coherence with the findings obtained by other methods. So, correlating volumetric MR data and MEG data of probable $\mathrm{AD}$ patients and a control group, they report that the higher the number of low frequency activity sources in left temporal and parietal areas, the higher the atrophy in the mesial aspects of the left temporal lobe. They conclude by proposing that "MTL atrophy and temporoparietal hypofunctioning may be considered complementary pictures of the same involutive process," but more than that, they reinforce the role of MEG as an important tool for a complementary techniques approach, overcoming in particular several EEG limitations concerning its precision. Several others works have directly addressed the importance of the concurrent validity of the various measurements. For instance, one of the first works to our knowledge specifically addressing the spatio-temporal patterns of brain magnetic activity in $\mathrm{AD}$, in a memory task, not only showed that this pattern was different among AD and elderly controls, with AD patients displaying reduced activation in temporal and parietal areas between 400 and $700 \mathrm{~ms}$ in late phases of stimuli processing, but, more importantly, the number of activity sources in the left parietal areas was a predictor of the scores on the cognitive and functional measurements that were used (Maestú, 2001). Subsequent work with the same subjects specifically examined the complementarily between functional measurements obtained with MEG and MRI volumetric measurements and neuropsychological data (Maestú, 2003). They found, among other results, that the degree of left hippocampal atrophy strongly correlates with the activation of left temporal areas, corroborating the existence of a relation between the structural integrity of the mesial temporal cortices, i.e. degree of atrophy, and the functional status of temporal and parietal regions i.e. lack of late brain magnetic activity. In a later study a specific pattern of changes was shown in resting state functional connectivity in AD patients. Synchronization likelihood (SL) was increased in the theta band over the central and parietal areas and in the beta band over the parietal and occipital areas. Coherence showed a similar pattern of parieto-occipital increase in $\mathrm{AD}$ in alpha 2, beta, and gamma bands. SL decreased in the alpha 1 band for long distance intrahemispheric sensor pairs, and both SL and coherence were decreased in the beta band for long distance frontal temporal parietal and short distance left temporal sensor pairs (Stam, 2006).

MEG seems also to be able to distinguish between AD and major depression in old people, with no differences being found between normal control subjects and elderly depressed 
patients in a working memory task. This reinforces the claim that memory deficits in depressed patients are more closely linked to emotional factors than substantial differences in neuronal activation. The fact that the AD group in this study displayed the expected pattern with a small number of activity sources in the left temporal region when compared with the other participants (with or without depression) reinforces this interpretation, as well as the claim that MEG has the potential to be useful in diagnosing AD (Maestú et al., 2004). Generally speaking, although it is not a very old technique, when MEG started to be used with $\mathrm{AD}$ patients it soon showed a promising potential to identify these patients and characterize their brain dynamics. In a recent review, Stam identifies the different contributions of spectral analysis, source analysis, task data, nonlinear analysis, and functional connectivity, concluding that since the first studies in 2000 MEG studies in AD have increased rapidly. The author suggests that there is now strong evidence that AD is characterized by a general slowing of background activity, reflected by increased power in delta and theta bands as well as a decreased power of alpha and beta bands, among other differences (Stam, 2010). The same author emphasizes that, despite improvements in the understanding of oscillatory and synchronized brain activity that can help to study disturbances such as $\mathrm{AD}$, as a large-scale network it is still lacking a proper theoretical framework to deal with the data obtained using these advanced techniques, proposing that focus should be placed instead on the small-world network, a combination of high clustering and short communication pathways, as one possible framework for the advancement of the study of AD.

However, MEG may have an important role not only in characterizing AD, but also in detecting the neuropathological process prior to its clinical manifestations. This necessity is acutely felt, since, as stated by several authors, there is a general agreement that the treatment strategies and interventions, ranging from cognitive stimulation to daily life changes as well as pharmacological approaches, should be as precocious as possible in order to maximize the impact of several groups of strategies in the delay of the symptoms and progression. However, that not only requires methodological and technical advancements but also conceptual ones. In fact, although it is also well known that pathological processes underlying Alzheimer's clinical onset start several years before, there are several difficulties in identifying subclinical Alzheimer's patients.

One of the most successful approaches in identifying these subjects, and one that is used in several clinical, behavioral, and neuroimaging studies, is the concept of Mild Cognitive Impairment (MCI). MCI patients are subjects who present memory impairment beyond that expected for age and education, yet are not demented, and who are at increased risk of progressing to AD (Petersen et al., 1999). In subsequent work it was pointed out that the construct of $\mathrm{MCI}$, with its subtypes, namely the amnestic subtype, has evolved to capture what can be seen as a pre-dementia phase of cognitive dysfunction (Gauthier et al., 2006). However, because many of them will not advance to AD, it may not be accurate to label participants at the aMCI stage as having AD or even prodromal AD (Petersen et al., 2009). There are still important sources of variability in MCI studies, such as source of the participants, clinical heterogeneity, neuropsychological normative data, etc.; however, the concept has been important because among other reasons it provides a framework for imaging and biomarkers, better characterizes those who will progress to AD, helps to identify risk factors, and increases predictability (Petersen et al., 2009). However, although there is great interest in adding biomarkers to the confirmation of an AD 
diagnosis, the final diagnosis of definite $\mathrm{AD}$ is still reliant on neuropathological examination (Mirra, 1991).

Nevertheless, despite the aforementioned sources of variability in MCI patients, the sensitivity of MEG in detecting features related to preclinical changes has been assessed in several previous studies. In a study comparing MCI patients and 10 normal controls assessed by means of MEG, the time course and the loci of brain activity in a deep encoding (semantic) and shallow encoding (nonsemantic) condition followed by a recognition task of previously presented words (Puregger et al., 2003), the mean recognition performances of $\mathrm{MCI}$ and controls were similar. Normal controls displayed no differences in the magnetic field distribution related to both encoding conditions. However, in the MCI the shallow encoding was associated with higher brain activity, mainly over left temporal and left frontal sensors rather than in the deep encoding condition. According to the authors, the results demonstrate that MCI patients showed significantly higher brain activity during shallow word encoding than deep word encoding between 250-450 ms after stimuli onset in the left medial temporal brain area. For the authors, this means that shallow encoding condition distinguishes neuropshysiological aspects of the groups, suggesting that nonsemantic new information may have this increased activation in MCI as a result of a compensatory process. It is worthwhile to note that all subjects displayed better performance after deep encoding (semantic), as predicted by the depth of processing background. Specifically investigating the power profiles and distribution of oscillatory sources of spontaneous MEG activity in MCI, and trying to gain advantage from the fact that it is a technique that has a better spatial resolution both at the sensor and source level compared to EEG, another study showed that the cerebral source of alpha activity is not changed in MCI when compared to normal controls (Osipova et al., 2006b). Subsequent work, clearly assuming that MCI patients represent an intermediate state between normal aging and dementia, tried to capitalize on MEG advantage for the acquisition of temporal courses of activation over high spatial resolution techniques, and tried to investigate the existence of a spatio-temporal memory profile that could discriminate between MCI and age-matched normal controls in a memory task. It was shown that the MCI subjects evidenced time-modulated increased activity in the ventral pathway during performance of a memory task, which was interpreted as a compensatory mechanism that allowed MCI patients to achieve behavioral results similar to the normal group (Maestú, 2008). Analysis of functional networks could also contribute to early detection of both MCI and AD. It seems that, in order to compensate for the loss of the segregation and integration balance, MCI subjects tend to increase their long-range synchronization, which could be underlying the increased blood flow showed in fMRI studies during memory tasks (Buldu et al., 2001). A network analysis, based on graph theory, revealed an important piece of information. Although MCI participants showed higher synchronization values than controls, they showed lower clustering values as well as higher values on the outreach parameters (physical distance between sensors), which in combination with other parameters is an indication of a loss of small-world architecture leading the MCIs to a more random structure. These findings argue against the compensatory hypothesis in the sense that the reorganized network does not lead to greater efficiency for information processing. In fact a more random network, compared to the controls, revealed a clear inefficiency in information processing even in the presence of higher synchronization, which can be recognized as spurious mechanisms (Bajo et al., JAD, 2010) 
In another study, the MEG scans of an initially normal elderly group (according to a careful neuropsychological, psychological, and medical evaluation) were obtained for a memory task. In a two-year follow up, when all the evaluation protocols were repeated, it was possible to identify decliners and non-decliners. When analyzing whether there were differences among early brain magnetic profiles between those two groups it was shown that brain activity at late latencies increased in those $\mathrm{MCI}$ patients that developed AD in comparison to non-decliners (Maestú et al, 2011), reinforcing MEG potential for the early prediction of dementia before symptoms are so clear that a conventional prognosis can be made. The same use of MEG as a possible method to estimate the risk of developing AD was assessed with $\mathrm{AD}$ patients, MCI patients, and healthy elderly controls when MEG recordings were obtained during a 5-minute resting period. Their results showed that high left parietal a dipole density is a possible marker of the risk of converting from MCI to AD within two years and, according to the authors, corroborated the interpretation that there is not a clear transition between MCI and AD (Fernandez, 2006).

Another important scenario where MEG may be an important tool is helping to clarify the clinical significance of subjective memory complaints (SMC), which is still a subject of debate. In a recent study, Maestu et al. (2011) specifically addressed this issue by comparing brain magnetic activity in three groups of elderly subjects: volunteers with SMC (memory complaints with a normal score in objective memory tasks), $\mathrm{MCI}$ (objective and subjective memory impairments), and a third group of normal subjects (neither objective nor subjective memory impairments). MEG scans were obtained in the context of a letter recognition task. When comparing the SMC and the MCI group no significant differences in brain activity were found. This is attributed to one of two possible scenarios: assuming that that SMC and $\mathrm{MCI}$ arise from similar neurophysiologic changes, the results may reflect lack of sensitivity of cognitive tests to detect the memory impairment in SMC. Alternatively, assuming that SMC and MCI have different etiologies, MEG may not be sensitive enough. The comparison between the SMC and the control group showed higher activation for the SMC in ventral posterior regions bilaterally and the dorsal pathway bilaterally between 200 and $900 \mathrm{~ms}$. When comparing MCI with the control group, they displayed greater activation, again between 200 and $900 \mathrm{~ms}$, mainly in areas similar to those found in previous work (Maestu et al., 2008); particularly in the ventral pathway (inferior parietal lobe), temporal lobe, and ventral prefrontal region. For late latency windows a bilateral increase in activity was also found in the dorsolateral prefrontal cortex. In a broad interpretation the bilateral activation is attributed to a compensatory mechanism in SMC and $\mathrm{MCI}$, similar to that proposed by the HAROLD (Hemispheric asymmetry in older adults) Model (Cabeza, 2002). Generally speaking, these results reinforce MEG ability to identify physiological differences between healthy elders and elders with complaints. Recognizing the necessity of further research, namely longitudinal research, for the authors the results suggest that SMC and MCI can be two stages in a cognitive continuum. In another study of SMC with MEG by the same research group, and with the same three groups of carefully selected subjects, i.e. SMC, MCI, and normals, and the same task, the functional connectivity (which, as stated previously, is regarded simply as a measure of the statistical interdependencies between two brain signals and seems to relate to the brain's ability to communicate information between brain regions) was specifically addressed, trying to determine if functional connectivity was, or was not, spared in the subjects with no objective memory deficit (Bajo et al., 2011). They found different profiles of functional connectivity, suggesting that the SMC profile, in comparison 
with the MCI subjects, was very similar to the one displayed when MCI and controls are compared. They point out that, taking as a standard the functional connectivity of normal elderly subjects, SMC subjects showed a hypo-synchronization of their memory-related networks, while MCI subjects showed an increased synchronization as a result of a compensatory process and as a means to increase communication; finally with AD this compensatory mechanism is no longer available. The point here is that the use of MEG can help us to describe profiles of prediction no longer only from $\mathrm{MCI}$ to $\mathrm{AD}$, but also from SMC to MCI. Additionally, the number of activity sources on the left MTL at the late latency window differed between those control subjects that subsequently developed $\mathrm{MCI}$ and those that did not (Maestú, 2006).

In a recent review, Zamrini et al. (2011) state that "biomarkers measured at the time of AD are excellent in predicting the presence of pathology at the time of autopsy, 8-10 years later. However they are less efficient at detecting the presence of pathology prior to the onset of clinical symptoms .... It seems necessary to have a biomarker that (1) measures neuronal activity directly, (2) has good temporal and spatial resolution, and (3) is able to evaluate functional networks and the associated neuronal code (i.e. oscillatory activity)." The same authors point out that electrophysiological / biomagnetic changes in the brain were not sufficiently explored as biomarkers bearing the potential of being the best indicator of the earliest functional changes secondary to the neuropathological process but prior to the clinical onset, and specifically discuss the MEG advantages over other AD biomarkers, namely EEG and $f$ MRI.

\section{Language and MEG}

Language is a distinctive function of human cognition, of extreme importance. Therefore, and trying to prevent the deleterious consequences of brain surgery, preoperative determination of language areas is a long recognized clinical application of neuroimaging of cognition and several and important MEG studies were done with this purpose. Since the first language MEG studies it was recognized that, since MEG information's can be used in surgical planning it is of extreme importance to establish its capacity to reveal the location of brain areas that show activity uniquely related to key language functions.

Language requires a distributed network, that was first addressed by lesions studies, wich comprises, among others, the classical language systems as well as other areas related to other involved cognitive functions. To use MEG to study language, as with other imaging techniques, we need well designed tasks in order to activate language areas. Besides specific language processes that include phonological, lexical, syntactic, and semantic processing, concurrent cognitive processes, for example concurrent memory processes, occur with any language paradigm. As previously stated, there are no pure tasks - and language paradigms are no exception.

MEG temporal resolution can also be used to better understand language. In an important MEG study where right-handed normal subjects where scanned during a memory recognition task for words in a visual and in an auditory task, the spatial outline of brain activation observed was in accord with previous findings using a variety of methodologies that suggest that temporoparietal and basal temporal areas, predominantly in the left hemisphere, are involved in reading. In addition they obtained evidence for activation in medial temporal areas consistent with previous findings implicating the hippocampus and 
the entorhinal cortex in memory function. They also took advantage of the unique spatiotemporal resolution of MEG to obtain information regarding the relative timing of association in these areas, showing that occipital lobe activity, generally associated with primary visual processing, was present in all the participants and was solved $100 \mathrm{~ms}$ after stimulus onset. As expected, the activity was bilateral - basal temporal areas next became active around 100 to $200 \mathrm{~ms}$ and were resolving by approximately $350 \mathrm{~ms}$ - and seemed to be specific to visually presented verbal stimuli. Temporoparietal areas of the dominant hemisphere are involved in a variety of language tasks, and activation of these areas was consistently observed after the activity in the basal temporal areas began to resolve. Activity in the medial temporal areas was concurrent with that in the temporoparietal areas. These results indicate a consistent temporal relationship between activity in the ventral pathway and temporoparietal cortex that supports a hypothesis regarding the functional specialization of these areas for different aspects of reading. To the authors these data suggested that MEG, by capturing the temporal as well as the spatial aspects of task-specific brain activity, had a promising role as an adjunct to other functional imaging modalities, serving to complement and extend their findings, particularly in language mapping (Breier et al., 1998). Other results reinforced the MEG role as a promising tool for identifying brain regions involved in the analysis of linguistic stimuli, in addition to the visual encoding of stimulus features (Simos et al., 1998). The number of left sources was greater than the number of right hemisphere sources in both tasks. In the word tasks the vast majority of sources were localized in temporal and temporo-parietal areas during latter portions of the waveform. In another study (Breier et. al, 1999) with epilepsy patients, an excellent agreement was found between the data obtained with magnetic source imaging and Wada indices.

A very important consideration is that MEG allows the component of sensory input to be excluded from the process. The temporal discrimination of MEG also allows us to study early components. Walla et al. (1999) confirmed that cognitive processes within word recognition tasks can be associated with different brain activities as early as under $200 \mathrm{~ms}$ after the stimulus onset, providing a distinction between the processes of encoding and the processes of retrieval attempts of verbal information. Because of its temporal resolution it can be used in studies specifically comparing early and late theories. For instance, to shed light on the time course of processing in visual word recognition, visual recognition of heteronymous- phonological and semantically distinct words that share a common orthography, providing a unique opportunity to distinguish between lexical and word form properties, were examined with MEG, finding results that suggest that true lexical access does not occur prior to $200 \mathrm{~ms}$, but rather at a latter stage of processing (Solomyak \& Marantz, 2009). A covert name and covert reading task concluded that MEG is a valuable tool to investigate both the time course of speech-evoked cortex activation as well as the localization of the main speech areas (Kober et al., 2001). The N400 component has also been addressed with MEG. This is an EEG-evoked response to the presentation of words and other meaningful stimuli that appear as a negative deflection in the ERP waveform, finding results that support a view in which the N400 effect observed in ERP reflects facilitated access of stored information rather than relative difficulty of semantic integration, "renewing the hope of a true top down effect on access" (Lau et al., 2009).

Several other works specifically addressed the determination of the language dominant hemisphere, which is critical for many clinical applications. But it is not an easy task to find 
a comprehensive protocol. In 2001, Breier et al. evaluated the validity of data derived from magnetic source imaging regarding hemispheric dominance for language in children and adolescents with intractable seizure disorders by a comparison with results of the intracarotid amobarbital procedure (IAP) showing that the laterality indices obtained by both procedures were highly correlated. They also found that independent clinical judgments based on both procedures to identify the lateralization of language function in individual patients were highly correlated. A 2002 study (Maestu et al., 2002) of Spanishspeaking patients with intractable epilepsy compared MEG data with IAP (intracarotid amytal procedure; see Maestu et al., Brain and Language, 2004 for a comparison with electrocortical stimulation). The results obtained closely parallel the original findings with English speakers and seem to support the cross-language generalization of the protocol used. This receives further support by the anatomical location of activity that shows the highest correlation with IAP data. To the authors these results confirmed the impression that these procedures are easy to implement, reliable, and valid, and may be used routinely to provide clinical information to increase the efficiency of pre-surgical evaluation. However, it is interesting to note that these patterns seem not to generalize for Chinese speakers (Valaki et al., 2004).

A 2009 paper by Frye et al. reviewed the functional neuroimaging of language using MEG and its importance as a functional neuroimaging technique for mapping the languagespecific cortex. Referring to the assessment of cerebral dominance, they suggest that the WADA procedure, while it is the most commonly used method for assessing cerebral dominance for language during pre-surgical evaluation of patients, has several limitations, namely concerning the invasiveness of the technique, but also its lack of regional specificity needed to determine the precise location and extent of the language-specific cortex. As these authors point out, many of their own works over the past decade, some of which are cited in this chapter, have included considerable efforts to develop a reliable MEG languagemapping protocol that could be used instead of more invasive procedures with no health risks, adaptable to test-retest reliability studies and able to be used in different tasks of extended duration. They point out that mapping of the language-specific cortex has been achieved using a variant of the Sternberg task for short-term memory known as the continuous recognition memory paradigm (CRM). Synthesizing several studies, they indicate that a stable feature of MEG-derived cortical activation maps in the context of CRM is a greater degree of activity in the left perysilvian region, in accordance with the expected left hemisphere dominance for receptive language in the majority of neurologically intact individuals. In a normative study of language profiles with MEG (Papanicolau et al., 2006) it was shown that brain activation profiles featured two components, an early and a late, but more importantly those features remained constant across task parameters, stimulus modality age, and gender of the participants, indicating that they constitute the basic aspects of the language perception mechanisms.

However, the main problem of MEG protocols in determining hemispheric dominance, when compared with Wada procedures, is that MEG protocols usually only test receptive language (reading and listening to single words), and there is a need to develop more robust paradigms capable of recording and modeling brain activity in anterior speech regions. There are already some expressive paradigms. In a study with a verb generation task it was possible to identify language lateralization by analyzing frequency specific spectral power changes (Fisher et al., 2008). Another MEG expressive language task successfully identified 
neural regions involved in language production showing high concordance with $f$ MRI verb generation to pictures and verb generation to words) but they all possess some caveats, namely, and according to the authors "the need for MEG users to be cognizant of the effects of task choice, threshold selection, MEG latency window and frequency band selections for data analyses" (Pang et al., 2011).

MEG has also proven its value with special populations. It was previously reported that several differences in brain functioning could be attributed to the absence of knowledge of orthography (Castro Caldas, 2004). Another study with MEG was done with subjects that learned to read and write during childhood (literates) and subjects who learned to read and write late in life (late literates). It was found that while subjects were visually recognizing previously presented words, late literates showed more late sources in right temporoparietal areas compared to controls, and controls showed more left inferior frontal sources. There is evidence that the right hemisphere plays a more important role in language processing in illiterate subjects. It is possible that the differences that arise after a $400 \mathrm{~ms}$ period can be attributed to a biased processing towards the right hemisphere. The interpretation proposed to explain the fact that late literate controls have fewer fonts in the left inferior frontal cortex is that control subjects have quicker access to the result of the visual decoding than late literates. Visual decoding gives access to the phonological form of the words, which is related to left IFG. Therefore this difference may reflect articulatory rehearsal. The general interpretation that we suggest for this group of results is that the process of learning to read and write during adulthood differs significantly from the process of learning to read and write in childhood (Castro Caldas et al., 2009). Ex-illiterate subjects also exhibited less brain functional asymmetry during an auditory recognition task with MEG (Silva Nunes et al., 2009) reinforcing the interpretation that education influences brain organization.

Other MEG studies involving children showed that compared to good readers, poor readers delayed and reduced left hemisphere activation to the most demanding phonological contrast and the overall sustained bilateral activation may reflect their greater difficulty with phonological processing (Wehner et al., 2007). As suggested by Frye et al. (2009), the bulk of MEG studies of children with dyslexia have shown that they exhibit a reduced ability to discriminate the phonological units of speech that contain fast auditory transients, such as syllables, displaying different spatio-temporal patterns of activation during the execution of reading tasks, and consistently exhibiting a reduced activation of the left posterior superiortemporal / supramarginal gyri, accompanied by hyper-activation of the righthomotopic regions and compensatory increases in pre-frontal activity. In a recent review of the contributions of MEG to reading it is pointed out that in order to read properly the brain is required to recruit distant cortical areas to function in highly complex and dynamic ways (Pammer, 2009), features that are well captured with EEG.

More recent work seems to be more theoretically driven and has isolated more specific aspects of language processing. For instance, Barca et al. (2011) studied Right Visual Field advantage in reading with MEG. RFV has been known about for more than 50 years, with the explanation that it has to do with language dominant hemisphere, which is for most right handers the left hemisphere. They found that activity in the speech motor area was lateralized to the left hemisphere and stronger for RVF than LVF words, which is interpreted as the proximal cause of the RFV advantage for the naming of written words. They also found that there were faster, stronger responses of the left midfusiform gyrus (Visual Word Form Area) to RVF than LVF words. Hirschfield et al. (2011) studied the 
effects of language comprehension on visual processing, taking advantage of MEG temporal resolution to conclude that while a perceptual match is detected a mere $120 \mathrm{~ms}$ after stimulus onset, overall semantic match between sentence content and pictures is indexed by a modulation of the N400. Another study looked at category-specific spatio-temporal representations, showing the importance of understanding not only the spatial but also the temporal representation of semantic categories using multivariate techniques (Chan et al., 2011).

Generally speaking, and despite the limitations raised; in terms of language study MEG is a valid tool for assessing cerebral dominance, elucidating temporal-spatial patterns of activation, and distinguishing between typical and atypical populations and between different components of language theories.

\section{Conclusion}

Because MEG is sensitive to the spatial and temporal properties of cortical signs, it can provide a valuable contribution to the study of cognition. As suggested by Rossini et al. (2007), "a major challenge of modern neuroscience is to identify patterns of neuronal activity underlying cognitive functions and to disentangle these actions from ongoing electromagnetic brain signals unrelated to task performance, background activity and function related activity," and there is now a growing awareness that a more complete understanding will only come through integration of different imaging technologies (Stufflebeam and Rosen, 2007). As an example, a recent study of visual short-term memory aimed to observe directly the relationship between the BOLD activation in intra-parietal and intra-occipital cortex and the electrophysiological component (Sustained Posterior Contralateral Negativity) and the Magnetoencephalographical marker (Sustained Posterior Contralateral Magnetic field) of the maintenance of information in visual short-term memory, specifically testing the hypothesis that both physiological markers (BOLD and SPCN/SPCM) reflect the same underlying neural processes. They tested the same subjects with fMRI, EGG, and MEG in very similar experiments of laterally-encoded stimuli in order to allow comparison between different techniques and with varying memory load (see Robitaille et al., 2010). However, contrary to what was expected the BOLD signal in IPS was the same in the left and right hemispheres regardless of the visual space where the stimuli was encoded; this is contrary to what happens with EEG and also, to a lesser extent, with MEG. The authors concluded that "despite the numerous advances in source localization of MEG signals, the use of multiple methods is still advised, along with careful interpretation of the results." Nevertheless, they point out that MEG was the only method capable of detecting cerebral activations bilaterally and contralaterally. Freeman et al. (2009) show that the brain as a thermodynamic system consumes energy at rates roughly tenfold greater than any other organ of comparable mass and that in the continuous interactions of excitatory and inhibitory currents in the same dendritic trees must at least partially cancel the extracellular electric and magnetic fields, but the metabolic needs must still be met for both forms of activation and for all frequency ranges. These authors point that, because inhibition is a process that requires expenditure of metabolic energy, activation and deactivation are radically different from excitation and inhibition. Multivariate approaches for characterizing and decoding local and distributed neuronal activity patterns in fMRi have been receiving increased attention in recent years. The integration of fMRI and EEG/MEG has received the most interest. MEG holds the promise of revealing dynamic connectivity since it is sensitive 
to transient neural activities occurring on the order of milliseconds. Cerebral functions are simultaneously localized and distributed (functional integration), which as pointed out above is important for the relevance of functional connectivity (Flecther et al., 1996). Also of importance is the investigation of the relationships between the functional connectivity as derived from fMRI data and from MEG/EEG data. Due to the different time scales and spatial resolutions of these two modalities there has not been a clearly established consistency between the results obtained with fMRI data and EEG/MEG alone.

As suggested by A. Ya. Kaplan et al. (2005), the search to understand how human beings create intentional behavior and how the mental world emerges within the human brain on the basis of neuronal activity inevitably leads researchers to study neuronal co-operation. Modern theoretical and experimental work suggests that the assemblies of couples and synchronously active neurons represent the most plausible candidates for the understanding of brain dynamics. Multimodal neuroimaging is becoming increasingly important (He and Liu, 2008).

As a final word, with all the recent and expected advances, there is no doubt that room for improvement still exists in functional neuroimaging. MEG can have an increased importance in the study of the functional connectivity, which is of pivotal importance in the study of cognition.

One of the aspects we consider to be of major importance is the study of individual activations. Although usually stated as a MEG advantage, individual differences are often "justified," perhaps because there is no integrated conceptual framework to accommodate the diversity. There is also, particularly for the study of cognition, room for improvement in the study of individual activations - once again, MEG can play an important role.

\section{References}

Aguirre, G. K. \& D'Esposito, M. Experimental design for brain fMRI. In Functional MRI ((2000). C.Moonen \& T. W. Bandettini (Eds.), Heidelberg: Springer-Verlag Berlin. pp. 369-380.

Anderson, K. L., Rajagovindan, R., Ghacibeh, G. A., Meador, K. J., \& Ding, M. (2010). Theta oscillations mediate interaction between prefrontal cortex and medial temporal lobe in human memor. Cerebral Cortex, Vol. 20, No. 7 (2010): p. 1604.

Babiloni, C., Cassetta, E., Chiovenda, P., Percio, C., Ercolani, M., Moretti, D., Moffa, F., Pasqualetti, P., Pizzella, V., Romani, G. L., Tecchio, F., Zappasodi, F., \& Rossini, P. M. (2005). Alpha rhythms in mild dements during visual delayed choice reaction time tasks: a MEG study. Brain Research Bulletin, Vol. 65, No. 6 (2005): pp. 457-470.

Bajo, R., Castellanos, N. P., López, M. E., Ruiz, J. M., Montejo, P., Montenegro, M., Llanero, M., Gil, P., Yubero, R., Baykova, E., Paul, N., Aurtenetxe, S., Del Pozo, F., \& Maestu, F. (2011). Early dysfunction of functional connectivity in healthy elderly with subjective memory complaints. Age (Dordr), (6 April 2011) [Epub ahead of print].

Bajo, R., Maestú, F., Nevado, A., Sancho, M., Gutiérrez, R., Campo, P., Castellanos, N. P., Gil, P., Moratti, S., Pereda, E., \& Del-Pozo, F. (2010). Functional connectivity in mild cognitive impairment during a memory task: Implications for the disconnection hypothesis. J Alzheimers Dis., Vol. 22, No. 1 (2010): pp. 183-193.

Bandettini, P. A. (2009). What's new in neuroimaging methods? Annals of the NY Academy of Sciences: The Year in Cognitive Neuroscience, Vol. 1156, No. 1 (2009): pp. 260-293. 
Barca, L., Cornelissen, P., Simpson, M., Urooj, U., Woods, W., \& Ellis, A. W. (2011). The neural basis of the right visual field advantage in reading: An MEG analysis using virtual electrodes. Brain Lang., Vol. 118, No. 3 (September 2011): pp. 53-71.

Berendse, H. W., Verbunt, J.P., Scheltens, P., van Dijk, B. W., \& Jonkman, E. J. (2000). Magnetoencephalographic analysis of cortical activity in Alzheimer's disease: a pilot study. Clinical Neurophysiology, Vol. 111, No. 4 (2000): pp. 604-12.

Breier, J. I., Simos, P. G., Zouridakis, G., \& Papanicolaou, A. C. (1998). Relative timing of neuronal activity in distinct temporal lobe areas during a recognition memory task for words. J Clin Exp Neuropsychol., Vol. 20, No. 6 (December 1998): pp. 782-90.

Breier, J. I., Simos, P. G., Wheless, J.W., Constantinou, J. E., Baumgartner, J. E., Venkataraman, V., \& Papanicolaou, A. C. (2001). Language dominance in children as determined by magnetic source imaging and the intracarotid amobarbital procedure: A comparison. J Child Neurol., Vol. 16, No. 2 (February 2001): pp. 124-30.

Breier, J. I., Simos, P. G., Zouridakis, G., Wheless, J. W., Willmore, L. J., Constantinou, J. E., Maggio, W. W., \& Papanicolaou, A. C. (1999). Language dominance determined by magnetic source imaging: a comparison with the Wada procedure. Neurology, Vol. 53, No. 5 (22 September 1999): pp. 938-45.

Buldú, J. M., Bajo, R., Maestú, F., Castellanos, N., Leyva, I., Gil, P., Sendiña-Nadal, I., Almendral, J. A., Nevado, A., Del-Pozo, F., \& Boccaletti, S. (2011). Reorganization of functional networks in mild cognitive impairment. PLoS One, Vol. 6, No. 5 (2011): e19584. Epub 2011 May 23.

Campo, P., Maestú, F., Ortiz, T., Capilla, A., Fernández, S., \& Fernández, A. (2005). Is medial temporal lobe activation specific for encoding long-term memories? NeuroImage, Vol. 25, No. 1 (March 2005): pp. 34-42.

Castro-Caldas, A., Nunes, M. V., Maestu, F., Ortiz, T., Simoes, R., Fernandes, R., de La Guia, E., Garcia, E., \& Goncalves, M. (2009). Learning orthography in adulthood: A magnetoencephalographic study. Journal of Neuropsychology, Vol. 3 (2009): pp. 1730.

Chan, A. M., Halgren, E., Marinkovic, K., \& Cash, S. S. (2011). Decoding word and categoryspecific spatiotemporal representations from MEG and EEG. Neuroimage, Vol. 54, No. 4 (14 February 2011): pp. 3028-39.

Deeny, S. P., Poeppel, D., Zimmerman, J. B., Roth, S. M., Brandauer, J., Witkowski, S., Hearn, J. W., Ludlow, A. T., Contreras-Vidal, J. L., Brandt, J., \& Hatfield, B. D. (2008). Exercise, APOE, and working memory: MEG and behavioral evidence for benefit of exercise in epsilon4 carriers. Biol Psychol, Vol. 78, No. 2 (May 2008): pp. 179-87.

Dixon R.A. e Nilsson L.G. (2004). Don't fence us in: Probing the frontiers of cognitive aging ( in New frontiers in cognitive aging, Eds. Dixon R.A., Backman L., Nilsson L.G. New York -Oxford University Press: pp 4-15

Dolan, R. J. (2008). Neuroimaging of cognition: Past, present, and future. Neuron, Vol. 60, No.3 (2008): pp. 496-502.

Donaldson, D. L. \& Buckner, R. L. (2001). Effective paradigm design. In P.Jezzard, P. M. Matthews, \& S. M. Smith (Eds.), Functional MRI: An introduction to methods. New York: Oxford University Press Inc.pp 177-195

Douw, L., Schoonheim, M. M., Landi, D., van der Meer, M. L., Geurts, J. J., Reijneveld, J. C., Klein, M., \& Stam, C. J. (2011). Cognition is related to resting-state small-world 
network topology: A magnetoencephalographic study. Neuroscience, Vol. 175 (February 2011): pp. 169-77.

Fernández, A., Arrazola, J., Maestú, F., Amo, C., Gil-Gregorio, P., Wienbruch, C., \& Ortiz, T. (2003). Correlations of hippocampal atrophy and focal low-frequency magnetic activity in Alzheimer's disease: Volumetric MR imaging-magnetoencephalographic study. AJNR Am J Neuroradiol, Vol. 24, No. 3 (March 2003): pp. 481-7.

Fernández, A., Hornero, R., Mayo, A., Poza, J., Gil-Gregorio, P., \& Ortiz, T. (2006). MEG spectral profile in Alzheimer's disease and mild cognitive impairment. Clinical Neurophysiology, Vol. 117, No. 2 (2006): pp. 306-314.

Fernández, A., Maestú, F., Amo, C., Gil, P., Fehr, T., Wienbruch, C., Rockstroh, B., Elbert, T., \& Ortiz, T. (2002). Focal temporoparietal slow activity in Alzheimer's disease revealed by magnetoencephalography. Biol Psychiatry, Vol. 52, No. 7 (1 October 2002): pp. 764-70.

Fernández, A., Turrero, A., Zuluaga, P., Gil, P., Maestú, F., Campo, P., \& Ortiz, T. (2006). Magnetoencephalographic parietal dipole density in mild cognitive impairment: Preliminary results of a method to estimate the risk of developing Alzheimer's Disease. Arch Neurol, Vol. 63, No. 3 (March 2006): pp. 427-430.

Fisher, A. E., Furlong, P. L., Seri, S., Adjamian, P., Witton, C., Baldeweg, T., Phillips, S., Walsh, R.,Houghton, J. M., \& Thai, N. J. (2008). Interhemispheric differences of spectral power in expressive language: A MEG study with clinical applications. International Journal of Psychophysiology, Vol. 68, No. 2 (2008): pp. 111-122.

Friston, K. J., \& Dolan, R. J. (2010). Computational and dynamic models in neuroimaging. Neuroimage, Vol. 52, No. 3 (September 2010): pp. 752-65.

Frye, R. E., Rezaie, R., \& Papanicolaou, A. C. (2009). Functional neuroimaging of language using magnetoencephalography. Physics of Life Reviews, Vol. 6, No. 1 (March 2009): pp. 1-10.

Gauthier, S., Reisberg, B., Zaudig, M., Petersen, R. C., Ritchie, K., Broich, K., Belleville, S., Brodaty, H., Bennett, D., Chertkow, H., Cummings, J. L., de Leon, M., Feldman, H., Ganguli, M., Hampel, H., Scheltens, P., Tierney, M. C., Whitehouse, P., \& Winblad, B. (2006). International Psychogeriatric Association Expert Conference on mild cognitive impairment: Mild cognitive impairment. Lancet, Vol. 367, No. 9518 (15 April 2006): pp. 1262-70.

Grummicha, P., Nimskya, C., Paulic, E., Buchfeldera, M., \& Ganslandta, M. (2006). Combining fMRI and MEG increases the reliability of presurgical language localization: A clinical study on the difference between and congruence of both modalities. NeuroImage, Vol. 32, No. 4.1 (October 2006): pp. 1793-1803.

Hämäläinen, M., \& Hari, R. (2002). Magnetoencephalographic characterization of dynamic brain activation: Basic principles and methods of data collection and source analysis, In: Brain Mapping: The Methods, A. W. Toga \& J. C. Mazziotta, Eds., Academic Press, Boston: pp. 227-253

Hari, R. (1993). Magnetoencephalography as a tool of clinical neurophysiology, In: Electroencephalography: Basic principles, clinical applications, and related fields, E. Niedermeyer \& F. L. da Silva, Eds., pp. 1035-1061, Williams \& Wilkins, Baltimore.

Hari, R., Levänen, S., \& Raij, T. (2000). Timing of human cortical functions during cognition: Role of MEG. Trends Cogn Sci, Vol. 4, No. 12 (1 December 2000): pp. 455-462. 
Hauk, O. (2004). Keep it simple: A case for using classical minimum norm estimation in the analysis of EEG and MEG data. Neuroimage, Vol. 21, No. 4 (April 2004): pp. 1612-21.

He, B., \& Liu, Z. (2008). Multimodal functional neuroimaging: Integrating functional MRI and EEG/MEG. IEEE reviews in biomedical engineering (2008): pp. 23-40.

Hirschfeld, G., Zwitserlood, P., \& Dobel, C. (2011). Effects of language comprehension on visual processing - MEG dissociates early perceptual and late N400 effects. Brain Lang, Vol. 116, No. 2 (2011): pp. 91-96.

Kaplan, A. Ya., Fingelkurts, A. A., Fingelkurts, A. A., Borisov, S. V., \& Darkhovskye, B. S. (2005). Nonstationary nature of the brain activity as revealed by EEG/MEG: Methodological, practical and conceptual challenges. Signal Processing, Vol. 85, No. 11 (November 2005): pp. 2190-2212.

Kim, M. S., Kim, J. S., \& Chung, C. K. (2008). Neural correlates of immediate and delayed word recognition memory: An MEG study. Brain Res, Vol. 1240 (November 2008): pp. 132-42.

Kober, H., Möller, M., Nimsky, C., Vieth, J., Fahlbusch, R., \& Ganslandt, O. (2001). New approach to localize speech relevant brain areas and hemispheric dominance using spatially filtered magnetoencephalography. Hum Brain Mapp, Vol. 14, No. 4 (December 2001): pp. 236-50.

Lau, E., Almeida, D., Hines, P. C., \& Poeppel, D. (2009). A lexical basis for N400 context effects:Evidence from MEG. Brain Lang, Vol. 111, No. 3 (December 2009): pp. 16172.

Maestú, F., Baykova, E., Ruiz, J. M., Montejo, P., Montenegro, M., Llanero, M., Solesio, E., Gil, P., Yubero, R., Paul, N., Pozo, F., \& Nevado, A. (2011). Increased biomagnetic activity in healthy elderly with subjective memory complaints. Clin Neurophysiol, Vol. 122, No. 3 (March 2011): pp. 499-505.

Maestú, F., Ortiz, T., Fernandez, A., Amo, C., Martin, P., Fernández, S., \& Sola, R. G. (2002). Spanish language mapping using MEG: a validation study. Neuroimage, Vol. 17, No. 3 (November 2002): pp. 1579-86.

Maestú, F., Fernandez, A., Simos, P., Lopez-Ibor, M., Campo, P., Criado, J., RodriguezPalanca, A., Ferre, F., Amo, C., \& Ortiz, T. (2004). Profiles of brain magnetic activity during a memory task in patients with Alzheimer's disease and in non-demented elderly subjects, with or without depression. J Neurol Neurosurg Psychiatry, Vol. 75, No. 8 (August 2004): pp. 1160-1162.

Maestú, F., Fernández, A., Simos, P. G., Gil-Gregorio, P., Amo, C., Rodriguez, R., Arrazola, J., \& Ortiz, T. (2001). Spatio-temporal patterns of brain magnetic activity during a memory task in Alzheimer's disease. Neuroreport, Vol. 12, No. 18 (21 December 2001): pp. 3917-22.

Maestú, F., García-Segura, J., Ortiz, T., Montoya, J., Fernández, A., Gil-Gregorio, P., Campo, P., Fernández, S., Viaño, J., \& Portera, A. (2005). Evidence of biochemical and biomagnetic interactions in Alzheimer's disease: An MEG and MR spectroscopy study. Dement Geriatr Cogn Disord, Vol. 20, No. 2-3 (2005): pp. 145-52.

Maestú, F., Arrazola, J., Fernández, A., Simos, P. G., Amo, C., Gil-Gregorio, P., Fernandez, A., Papanicolaou, A. C., \& Ortiz, T. (2003). Do cognitive patterns of brain magnetic activity correlate with hippocampal atrophy in Alzheimer's disease? J Neurol Neurosurg Psychiatry, Vol. 74 (2003): pp. 208-12. 
Maestú, F., Campo, P., Gil-Gregorio, P., Fernández, S., Fernández, A., \& Ortiz, T. (2006). Medial temporal lobe neuromagnetic hypoactivation and risk for developing cognitive decline in elderly population: A 2-year follow-up study. Neurobiol Aging, Vol. 27, No. 1 (January 2006): pp. 32-37.

Maestú, F., Campo, P., Del Río, D., Moratti, S., Gil-Gregorio, P., Fernández, A., Capilla, A., \& Ortiz, T. (2008). Increased biomagnetic activity in the ventral pathway in mild cognitive impairment. Clinical Neurophysiology, Vol. 85, No. 11 (2008): pp. 2190-2212.

Mirra, S. S., Heyman, A., McKeel, D., Sumi, S. M., Crain, B. J., Brownlee, L. M., Vogel, F. S., Hughes, J. P., van Belle, G., \& Berg, L. (1991). The Consortium to Establish a Registry for Alzheimer's Disease (CERAD). Part II. Standardization of the neuropathologic assessment of Alzheimer's disease. Neurology, Vol. 41, No. 4 (April 1991): pp. 479-86.

Moran, R. J., Campo, P., Maestu, F., Reilly, R. B., Dolan, R. J., \& Strange, B. A. (2010). Peak frequency in the theta and alpha bands correlates with human working memory capacity. Front Hum Neurosci, Vol. 11, No. 4 (November 2010): p. 200.

Osipovaa, D., Ahveninenb, J., Jensene, O., Ylikoskif, A., \& Pekkonena, E. (2005). Altered generation of spontaneous oscillations in Alzheimer's disease. NeuroImage, Vol. 27, No. 4 (2005): pp. 835-41.

Osipovaa, D., Rantanend, K., Ahveninene, J., Ylikoskid, R., Häppöläd, O., Strandbergf, T., \& Pekkonena, E. (2006). Source estimation of spontaneous MEG oscillations in mild cognitive impairment. Neuroscience Letters, Vol. 405, No. 1-2 (September 2006): pp. 57-61.

Osipovaa, D., Pekkonen, E., \& Ahveninen, J. (2006). Enhanced magnetic auditory steadystate response in early Alzheimer's disease. Clinical Neurophisiology, Vol. 117, No. 9 (October 2006): pp. 1990-95.

Pang, E. W., Wang, F., Malone, M., Kadis, D. S., \& Donner, E. J. (2011). Localization of Broca's area using verb generation tasks in the MEG: Validation against fMRI. Neuroscience Letters, Vol. 490, No. 3 (3 March 2011): pp. 215-19.

Papanicolaou, A. C. (1998). Fundamentals of Functional Brain Imaging: A guide to the methods and their applications to psychology and behavioral neurosciences. Swets \& Zeitlinger, Netherlands.

Papanicolaou, A. C., Pazo-Alvarez, P., Castillo, E. M., Billingsley-Marshall, R. L., Breier, J. I., Swank, P. R., Buchanan, S., McManis, M., Clear, T., \& Pássaro, A. D. (2006). Functional neuroimaging with MEG: Normative language profiles. NeuroImage, Vol. 33, No. 1 (October 2006): pp. 326-42.

Pasquale, F., Della Penna, S., Snyder, A. Z., Lewis, C., Mantini, D., Marzetti, L., Belardinelli, P., Ciancetta, L., Pizzella, V., Romani, G. L., \& Corbetta, M. (2010). Temporal dynamics of spontaneous MEG activity in brain networks. Proc Natl Acad Sci, Vol. 107, No. 13 (30 March 2010): pp. 6040-45.

Petersen, R. C., Roberts, R. O., Knopman, D. S., Boeve, B. F., Geda, Y. E., Ivnik, R. J., Smith, G. E., Jack, C. R., Jr. (2009). Mild cognitive impairment: Ten years later. Arch Neurol, Vol. 66, No. 12 (December 2009): pp. 1447-55.

Petersen, R. C., Smith, G. E., Waring, S. C., Ivnik, R. J., Tangalos, E. G., \& Kokmen, E. (1999). Mild cognitive impairment: Clinical characterization and outcome. Arch Neurol, Vol. 56, No. 3 (1999): pp. 303-8. 
Poza, J., Hornero, R., Abásolo, D., Fernández, A., \& García, M. (2007). Extraction of spectral based measures from MEG background oscillations in Alzheimer's disease. Med Eng Phys, Vol. 29, No. 10 (December 2007): pp. 1073-83.

Poza, J., Hornero, R., Abásolo, D., Fernández, A., \& Mayo, A. (2008). Evaluation of spectral ratio measures from spontaneous MEG recordings in patients with Alzheimer's disease. Computer Methods and Programs in Biomedicine, Vol. 90, No. 2 (2008): pp. 137-47.

Puligheddu, M., de Munck, J. C., Stam, C. J., Verbunt, J., de Jongh, A., van Dijk, B. W., \& Marrosu, F. (2005). Age distribution of MEG spontaneous theta activity in healthy subjects. Brain Topogr., Vol. 17, No. 3 (Spring 2005): pp. 165-75.

Pulvermüller, F., Shtyrov, Y., \& Ilmoniemi, R. (2003). Spatiotemporal dynamics of neural language processing: An MEG study using minimum-norm current estimates. Neuroimage, Vol. 20, No. 2 (October 2003): pp. 1020-5.

Püregger, E., Walla, P., Deecke, L., \& Dal-Bianco, P. (2003). Magnetoencephalographicfeatures related to mild cognitive impairment. Neuroimage, Vol. 20, No. 4 (December 2003): pp. 2235-44.

Raichle, M. (19939 Functional Neuroimaging: A Historical and Physiological Perspective in Handbook of Functional Neuroimaging of Cognition: Cabeza R. \& Kingstone A., Eds., pp. 3-26, MIT Press.

Robitaille, N., Marois, R., Todd, J., Grimault, S., Cheyne, D., \& Jolicoeur, P. (2010). Distinguishing between lateralized and nonlateralized brain activity associated with visual short-term memory: fMRI, MEG, and EEG evidence from the same observers. Neuroimage, Vol. 53, No. 4 (December 2010): pp. 1334-45.

Rossini, P. M., Rossi, S., Babiloni, C., \& Polich, J. (2007). Clinical neurophysiology of aging brain: From normal aging to neurodegeneration. Progress in Neurobiology, Vol. 83, No. 6 (December 2007): pp. 375-400.

Salmelin, R. (2007). Clinical neurophysiology of language: The MEG approach. Clinical Neurophysiology, Vol. 118, No. 2 (2007): pp. 237-54.

Silva Nunes, M. V., Castro-Caldas, A., Rio, D. D., Maestú, F., \& Ortiz, T. (2009). The exilliterate brain: the critical period, the cognitive reserve and the HAROLD model. Dement. Neuropsychol., Vol. 3, No. 3 (2009): pp. 222-27.

Simos, P. G., Breier, J. I., Zouridakis, G., \& Papanicolaou, A. C. (1998). Identification of language-specific brain activity using magnetoencephalography. J Clin Exp Neuropsychol., Vol. 20, No. 5 (October 1998): pp. 706-22.

Solomyak, O. \& Marantz, A. (2009). Lexical Access in Early Stages of Visual Word Processing: A Single-Trial Correlational MEG Study of Heteronym Recognition. Brain and Lang, Vol. 108, No. 3 (March 2009): pp. 191-96.

Stam, C. J., Jones, B. F., Manshanden, I., van Cappellen van Walsum, A. M., Montez, T., Verbunt, J. P., de Munck, J. C., van Dijk, B. W., Berendse, H. W., \& Scheltens, P. (2006). Magnetoencephalographic evaluation of resting-state functional connectivity in Alzheimer's disease. NeuroImage, Vol. 32, No. 3 (2006): pp. 1335-44.

Stam, C. J. (2010). Use of magnetoencephalography (MEG) to study functional brain networks in neurodegenerative disorders. Journal of the Neurological Sciences, Vol. 289, No. 15 (February 2010): pp. 128-34.

Stam, C. J. (2005). Nonlinear dynamical analysis of EEG and MEG: Review of an emerging field. Clin Neurophysiol., Vol. 116, No. 10 (October 2005): pp. 2266-2301. 
Stufflebeam, S. M., \& Rosen, B. R. (2007). Mapping cognitive function. Neuroimaging Clin N Am., Vol. 17, No. 4 (November 2007): pp. 469-84, viii-ix.

Tanaka, K., Kawakatsu, M., Fujiwara, K., Wang, G., \& Yunokuchi, K. (2003). Tokyo Denki Effects on MEG coherence with working memory load. The IEEE EMBS AsianPacific Conference on Biomedical Engineering 2003, pp. 334-35.

Valaki, C. E., Maestu, F., Simos, P. G., Zhang, W., Fernandez, A., Amo, C. M., Ortiz, T., \& Papanicolaou, A. C. (2004). Cortical organization for receptive language functions in Chinese, English, and Spanish: A cross-linguistic MEG study. Neuropsychologia, Vol. 42, No. 7 (2004): pp. 967-79.

Walla, P., Endl, W., Lindinger, G., Lalouschek, W., Deecke, L., \& Lang, W. (1999). Early occipito-parietal activity in a word recognition task: an EEG and MEG study. Clin Neurophysiol., Vol. 110, No. 8 (August 1999): pp. 1378-87.

Wehner, D. T., Ahlfors, S. P., \& Mody, M. (2007). Effects of phonological contrast on auditory word discrimination in children with and without reading disability: A magnetoencephalography (MEG) study. Neuropsychologia, Vol. 45, No. 14 (November 2007): pp. 3251-62.

Zamrini, E., Maestu, F., Pekkonen, E., Funke, M., Makela, J., Riley, M., Bajo, R., Sudre, G., Fernandez, A., Castellanos, N., Del Pozo, F., Stam, C. J., van Dijk, B. W., Bagic, A., \& Becker, J. T. (2011). Magnetoencephalography as a putative biomarker for Alzheimer's disease. Int J Alzheimers Dis. (10 April 2011): pp. 280-89. 


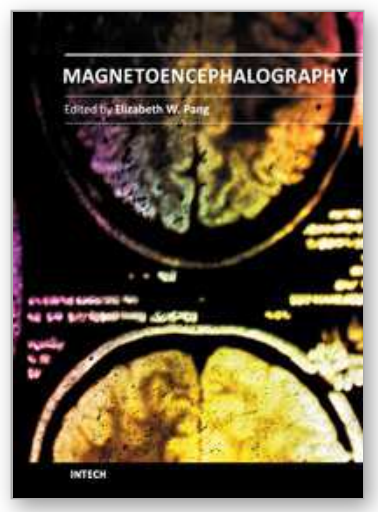

\author{
Magnetoencephalography \\ Edited by Dr. Elizabeth Pang
}

ISBN 978-953-307-255-5

Hard cover, 252 pages

Publisher InTech

Published online 30, November, 2011

Published in print edition November, 2011

This is a practical book on MEG that covers a wide range of topics. The book begins with a series of reviews on the use of MEG for clinical applications, the study of cognitive functions in various diseases, and one chapter focusing specifically on studies of memory with MEG. There are sections with chapters that describe source localization issues, the use of beamformers and dipole source methods, as well as phase-based analyses, and a step-by-step guide to using dipoles for epilepsy spike analyses. The book ends with a section describing new innovations in MEG systems, namely an on-line real-time MEG data acquisition system, novel applications for MEG research, and a proposal for a helium re-circulation system. With such breadth of topics, there will be a chapter that is of interest to every MEG researcher or clinician.

\title{
How to reference
}

In order to correctly reference this scholarly work, feel free to copy and paste the following:

M.V. Silva Nunes, F. Maestú and A. Castro Caldas (2011). The Role of MEG in Unveiling Cognition, Magnetoencephalography, Dr. Elizabeth Pang (Ed.), ISBN: 978-953-307-255-5, InTech, Available from: http://www.intechopen.com/books/magnetoencephalography/the-role-of-meg-in-unveiling-cognition

\section{INTECH}

open science | open minds

\section{InTech Europe}

University Campus STeP Ri

Slavka Krautzeka 83/A

51000 Rijeka, Croatia

Phone: +385 (51) 770447

Fax: +385 (51) 686166

www.intechopen.com

\section{InTech China}

Unit 405, Office Block, Hotel Equatorial Shanghai

No.65, Yan An Road (West), Shanghai, 200040, China

中国上海市延安西路65号上海国际贵都大饭店办公楼405单元

Phone: +86-21-62489820

Fax: $+86-21-62489821$ 
(C) 2011 The Author(s). Licensee IntechOpen. This is an open access article distributed under the terms of the Creative Commons Attribution 3.0 License, which permits unrestricted use, distribution, and reproduction in any medium, provided the original work is properly cited. 